\title{
A Robot and Control Algorithm That Can Synchronously Assist in Naturalistic Motion During Body-Weight-Supported Gait Training Following Neurologic Injury
}

\author{
Daisuke Aoyagi, Wade E. Ichinose, Susan J. Harkema, David J. Reinkensmeyer, Member, IEEE, and
} James E. Bobrow, Member, IEEE

\begin{abstract}
Locomotor training using body weight support on a treadmill and manual assistance is a promising rehabilitation technique following neurological injuries, such as spinal cord injury (SCI) and stroke. Previous robots that automate this technique impose constraints on naturalistic walking due to their kinematic structure, and are typically operated in a stiff mode, limiting the ability of the patient or human trainer to influence the stepping pattern. We developed a pneumatic gait training robot that allows for a full range of natural motion of the legs and pelvis during treadmill walking, and provides compliant assistance. However, we observed an unexpected consequence of the device's compliance: unimpaired and SCI individuals invariably began walking out-ofphase with the device. Thus, the robot perturbed rather than assisted stepping. To address this problem, we developed a novel algorithm that synchronizes the device in real-time to the actual motion of the individual by sensing the state error and adjusting the replay timing to reduce this error. This paper describes data from experiments with individuals with SCI that demonstrate the effectiveness of the synchronization algorithm, and the potential of the device for relieving the trainers of strenuous work while maintaining naturalistic stepping.
\end{abstract}

Index Terms-Backdrivable, gait rehabilitation, pneumatic, robot, spinal cord injury, stroke, synchronization.

\section{INTRODUCTION}

$\mathbf{L}$ OCOMOTOR training using body weight support on a treadmill (BWST) and manual assistance of the legs and the pelvis is an emerging technique for gait rehabilitation following neurological injuries, such as spinal cord injury (SCI) and stroke, that has shown promising results [1]-[6]. This method uses an overhead suspension system to unload the

Manuscript received September 30, 2006; revised March 19, 2007; accepted March 24, 2007. This work was supported in part by the National Institute of Standards and Technology (NIST) Advanced Technology Program (ATP) under Award 00-00-4906, in part by the National Institute on Disability and Rehabilitation Research (NIDRR) Rehabilitation Engineering Research Center on Spinal Cord Injury (H133E020732), and in part by a graduate fellowship from Parker Hannifin.

D. Aoyagi and W. E. Ichinose are with the Los Amigos Research and Education Institute, Downey, CA 90242 USA.

S. J. Harkema is with the University of Louisville, Frazier Rehab Institute, Louisville, KY 40202 USA.

D. J. Reinkensmeyer and J. E. Bobrow are with the University of California, Irvine, CA 92697 USA.

Color versions of one or more of the figures in this paper are available at http://ieeexplore.ieee.org.

Digital Object Identifier 10.1109/TNSRE.2007.903922
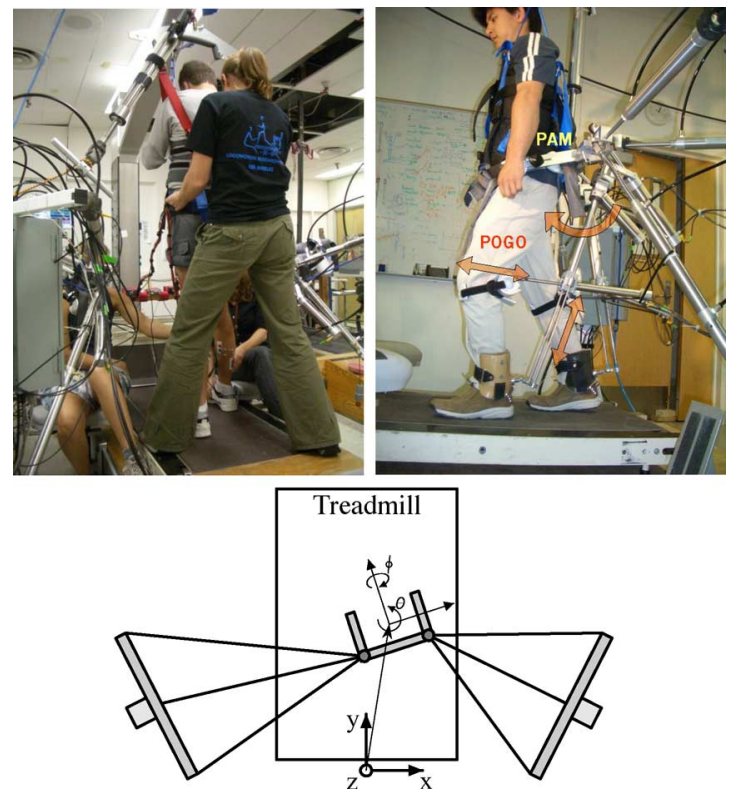

Fig. 1. Top left: Three trainers guide the legs and the torso of the patient suspended by the body weight support system on a treadmill. Top right: PAM and POGO are inherently compliant robotic devices that could act either in aid of the trainers, or in place of them if desired. Bottom: PAM has five actuated DOF, namely forward-and-back $(x)$, side-to-side $(y)$, up-and-down $(z)$ and pelvic rotation $(\theta)$ and obliquity $(\phi)$.

body weight of the patient as necessary, while trainers guide the legs and the torso of the patient through a gait-like motion on a treadmill. Typically, the trainers try to coordinate various tasks simultaneously: to prevent the knee from buckling during stance, to promote a smooth leg swing by adding momentum, to avoid dragging of the toe, to guide the timing and location of foot landing, and to encourage weight shifting through the pelvic motion. Although the BWS system relieves the trainers of the major labor of supporting and stabilizing the patient's whole body throughout a training session, the training procedure is still highly labor intensive, requiring three skilled trainers to assist the patient's legs and torso and to operate the treadmill and BWS system (Fig. 1). Also, the manual assistance provided can vary greatly between trainers and between training sessions [7]. Therefore, introduction of robotic devices into rehabilitation has several potential benefits. Robotic devices can provide measurements of actual kinematics and forces. They can potentially provide assistance to the trainers and the patient, allowing for 
more consistent therapy with longer and more frequent training sessions.

Several research groups have developed robotic devices for providing assistance during locomotor training. For example, the mechanized gait trainer (MGT) moves a patient's legs in a gait-like pattern by driving two foot plates connected to a double crank and rocker system that is actuated by a motor via a planetary gear system [8]. The Lokomat is a motorized exoskeleton that can drive hip and knee flexion through the four rotary joints driven by dc motors via precision ball screws [9].

Several joint motions that are natural to human gait are structurally constrained in these devices. For instance, once strapped into the Lokomat, patient's movements are restricted to the actuated four rotary degrees-of-freedom (DOF) of the legs and the straight vertical motion of the torso. The device does not allow for the leg swing out of the parasagittal plane, pelvic rotation, or lateral motion of the pelvis, even though pelvic motion plays an important role in locomotion, with the pelvis undergoing three translational displacements and three angular displacements during unconstrained normal locomotion [10]. In fact, during a typical BWS gait training session, a trainer assists the pelvic motion to promote proper weight shift and leg swing, while maintaining balance and stability [5]. The Lokomat developers have begun to address this issue by developing a version of the machine that allows hip abduction and adduction, but horizontal pelvic rotation is still not possible. In the case of the MGT, the ellipsoid-like trajectory of the foot plates is essentially fixed once the gears are set, although the velocity along the trajectory could potentially be changed according to, for example, the forces on the foot plates. As a result of such kinematic constraints, the dynamics experienced by the patient can be substantially different from that of an actual gait [11]. This may lead to an unnatural pattern of somatosensory input to the patient's nervous system, impairing the effectiveness of the therapy. Our concern is that the patient may relax his effort if the robot rigidly imposes a motion, and possibly learn to rely on it; the robot may become an analogy of training wheels that will not come off a bicycle.

Making robotic assistance compliant so that the patient sees and feels the results of a decreased effort is an approach toward encouraging patient effort. For example, the MIT-MANUS is one of the first robotic devices that employed the advantage of a light weight and compliant robot design with force controllability [12]. Having low intrinsic endpoint impedance with low inertia and friction (i.e., backdrivable), it can guide, perturb and, more importantly, not perturb the movement of the upper limb when the patient moves freely, while recording mechanical quantities such as the position, velocity, and forces. The ambulation-assisting robotic tool for human rehabilitation (ARTHuR) achieves backdrivability and force control capability with a two bar linkage mechanism and two linear motors, and can measure and manipulate the motion of either the ankle or the knee during stepping on a treadmill [13]. The Lokomat research group has also recently addressed the potential problem of rigid assistance by implementing impedance control that relies on sensing interaction force between the patient and the device, making it possible to provide compliant assistance to the legs [14], [15]. Likewise, the MGT group has designed a device (the "Haptic
Walker") with more DOF that relies on force feedback to accommodate more natural motion of the legs [16], [17].

The goal of the present project was to design a robot that could accommodate naturalistic motion of the patient including the pelvis, and coexist with human trainers at the legs and the pelvis. Our approach was not to start from a rigid robot and add compliance to it, but rather to start from an inherently compliant device and use it to apply forces only as needed. A key design goal was to not block the human trainers' direct physical interaction with the patient, and to allow the trainers to manually exert direct control over the patient's movement at any time as they see fit. Put another way, the goal was to create a compliant robot that could act either in aid of the trainers, or in place of them if desired.

In this paper, we describe the development of the pelvic assist manipulator (PAM), a device that can assist the pelvic motion during stepping using BWST, and the pneumatically operated gait orthosis (POGO), which is designed as an attachment to PAM. Because they are actuated by pneumatic cylinders, PAM and POGO are inherently compliant, yet are able to produce large force with relatively lightweight moving parts. Having six DOF, of which all except pelvic tilt are actuated, PAM allows unconstrained motion of the pelvis during gait. The rationale for omitting robotic control of pelvic tilt is that it is mostly supported by the BWS system, and human trainers do not typically apply torque in that direction. POGO is designed to provide assistance for the leg swing, and to prevent buckling of the knees during the stance phase, without imposing abnormal constraints on the naturalistic walking motion.

Controlling these compliant pneumatic actuators for gait training required development of new control techniques. Unlike typical industrial robot manipulators, we do not pursue precise position control, for which a stiff structure would be better suited. Instead, what we seek is a good force modulation. Previously, we developed a nonlinear controller to achieve force tracking with the pneumatic actuators, including backdrivability (i.e., zero force control), and constructed an impedance-type position controller to demonstrate PAM's ability as a teach-and-replay device that can drive the pelvis toward a reference trajectory [18]. However, during our initial testing with this controller with unimpaired individuals, we observed an unexpected consequence of the compliant assistance; they invariably began walking out-of-phase with the robot. In particular, we observed a phenomenon very similar to that of "beats" that appears when two waveforms at slightly different frequencies interfere with each other. When the periodic robotic assistance was applied onto the periodic walking pattern of the individual, the pair of coupled "oscillators" (i.e., the human and the robot) generated a sort of ebb and flow of the amplitude of the periodic motion. Even though the period of the robotic assistance was set to the average step period measured from the same individual beforehand, the oscillators were not always synchronized to each other, and the difference of the timing caused an apparently large tracking error, which resulted in "corrective" forces that actually perturbed rather than assisted the individual's intended motion. Such forces in turn influenced step timing and movement of the individual, who usually reacted by taking shorter and quicker steps, resulting in a 
further mismatch in the timing. This type of desynchronization problem appears likely to occur whenever a compliant robot assists in a periodic motion made by a human.

To address this problem, we previously proposed a feedback control algorithm that adjusts the replay speed of the reference trajectory based on the step timing measured by threshold detection on the footswitch signal [19]. This algorithm was effective in synchronizing the robot with unimpaired individuals, even when they deliberately changed their step size and period. However, it was cumbersome to hook up the footswitches required for this algorithm, and footswitches can sometimes provide unreliable signals, which could degrade the operation of the algorithm. Aiming to eliminate the use of footswitches and to achieve smoother and more robust synchronization performance, we developed an algorithm that continuously estimates the subject's phase in the gait cycle based on the position and velocity measured by the device. We then modified the synchronization algorithm so that it can work in a continuous manner (i.e., adjusting timing within a step) as opposed to the discrete operation (i.e., adjusting timing between steps) of the footswitch-based algorithm. In this paper, we describe the algorithm, and report the first experiments with individuals with SCI that demonstrate the capability of the device and control algorithm to assist in gait training.

\section{METHODS}

\section{A. Apparatus}

1) PAM: The PAM can be divided into a pair of subrobots, each having three pneumatic cylinders arranged in a tripod configuration supported by a height-adjustable $T$-shaped pillar via universal joints (Fig. 1). This design allows unconstrained range-of-motion of the pelvis during normal gait, and provides room for arm swing and an unobstructed field-of-view for the individual. The two subrobots are placed at an angle to give the trainers access to the individual from the sides and from behind. They can be detached and separated to make sufficient spacing for entry of an individual on a wheelchair.

A custom-designed linkage allows the axes of the three cylinders to intersect at a point. The pair of tripods attach to the back of a width-adjustable belt piece worn by an individual via ball joints. The complete PAM system, consisting of the two actuated tripods coupled together with the belt piece, has five actuated DOF, namely three translations (side-to-side, forward-and-back, up-and-down) and two rotations (pelvic rotation and obliquity) (Fig. 1). The remaining passive DOF (pelvic tilt) is not measured or controlled, but the overhead BWS system keeps it stabilized. Here, we define pelvic angles as follows. The pelvic rotation is the angle that the projection of the medio-lateral axis of the pelvis onto the horizontal plane makes with the treadmill-based medio-lateral axis. It roughly refers to "swivel" in the horizontal plane. Obliquity is the angle of rotation of the medio-lateral axis of the pelvis out of the horizontal plane. Tilt is the angle of rotation about the medio-lateral axis of the pelvis [20].

For enhanced safety, an additional hard-stop structure mechanically prohibits extreme rotations. The hard-stop, hanging via a universal joint from the overhead frame, can swing like a pendulum and vary its length to allow free translations within the workspace, but limits the angular motions within $\pm 40^{\circ}$ (pelvic rotation) and $\pm 15^{\circ}$ (obliquity), with these amounts being adjustable. The default limits are selected to accept somewhat exaggerated motion that the trainers often induce during training, but to prohibit extreme angles that are possibly too uncomfortable or dangerous. The translational workspace is limited by the stroke lengths of the cylinders (roughly $25 \times$ $25 \mathrm{~cm}$ in horizontal plane and $20 \mathrm{~cm}$ vertical) [18]. A separate overhead BWS system (Robomedica Inc., Irvine, CA) unloads the patient by controlling the tension of the wire that suspends the individual.

The pneumatic cylinders (Bimba Manufacturing Company, Monee, IL) have a built-in linear potentiometer. From the measured cylinder lengths, we solve the kinematics to find the position of the belt worn by an individual. We compute the velocity from the position signals by taking a numerical derivative and filtering. We measure the cylinder pressures, from which we estimate the net force output of the cylinders. With the pneumatic actuators, PAM is capable of producing large forces at a relatively low cost of approximately $\$ 1000$ per DOF, including cylinder, servovalves (Festo Corporation, Hauppauge, NY), and circuitry costs. The compliant characteristic of the air is well suited for our design criteria, and the actual moving parts can be made light.

We set the supply pressure and flow rate to physically limit the maximum force and power output of the actuators. At a $300 \mathrm{kPa}$ (44 PSI) supply pressure, PAM can generate roughly $600 \mathrm{~N}$ $(135 \mathrm{lb})$ of force in the horizontal plane and $300 \mathrm{~N}(67 \mathrm{lb})$ vertically. We can achieve sufficiently good force control (approximately $5-10 \mathrm{~Hz}$ bandwidth) by applying the nonlinear forcetracking controller we have developed, which controls the gas flow into and out of each chamber of a cylinder [21]. Backdrivability is achieved by setting the desired force to zero. In this "backdrive-mode," PAM basically yields to the forces applied to it.

All cylinders can be vented at any time by manual switches held by an operator and the individual stepping in the robot to render the robot mechanically passive. If any fault condition, including inconsistent kinematics (there are six cylinders sensing five DOF, providing a redundant check on kinematics), abnormally high velocity and large force tracking error, is detected, or if the watchdog timer detects a computer malfunction, all cylinders are fully vented through pressure relief valves. We use Matlab Simulink and xPC Target (Mathworks Inc., Natick, MA) for implementing the real-time control tasks at a sampling rate of $500 \mathrm{~Hz}$.

2) POGO: The POGO is an attachment to PAM. Having two actuated DOF per side (the hip and knee) with force controllability, it provides power assistance for leg swing and prevents knee buckling during stance. In addition to the actuated DOF, it has passive DOF, allowing for naturalistic leg motion. For instance, leg swing out of the parasagittal plane is allowed (hip abduction/adduction), and the attachment braces connected via a universal joint provide additional passive DOF. Due to its light weight and compliance, POGO is worn by the individual, with his or her legs actually serving as a part of the kinematic structure. We designed the attachment braces so that they imitate the 
TABLE I

PD CONTROLLER GAINS FOR PAM AND POGO

\begin{tabular}{cc||c|c}
\hline & & $G_{P}$ & $G_{D}$ \\
\hline \hline \multirow{4}{*}{ PAM } & Lateral & $2800[\mathrm{~N} / \mathrm{m}]$ & $150[\mathrm{~N} \cdot \mathrm{s} / \mathrm{m}]$ \\
\cline { 2 - 4 } & Forward & $3000[\mathrm{~N} / \mathrm{m}]$ & $150[\mathrm{~N} \cdot \mathrm{s} / \mathrm{m}]$ \\
\cline { 2 - 4 } & Upward & $3000[\mathrm{~N} / \mathrm{m}]$ & $150[\mathrm{~N} \cdot \mathrm{s} / \mathrm{m}]$ \\
\cline { 2 - 4 } & Rotation & $200[\mathrm{Nm} / \mathrm{rad}]$ & $20[\mathrm{Nm} \cdot \mathrm{s} / \mathrm{rad}]$ \\
\cline { 2 - 4 } & Obliquity & $200[\mathrm{Nm} / \mathrm{rad}]$ & $20[\mathrm{Nm} \cdot \mathrm{s} / \mathrm{rad}]$ \\
\hline \multirow{3}{*}{ POGO } & Left Hip & $80[\mathrm{Nm} / \mathrm{rad}]$ & $5[\mathrm{Nm} \cdot \mathrm{s} / \mathrm{rad}]$ \\
\cline { 2 - 4 } & Left Knee & $350[\mathrm{~N} / \mathrm{m}]$ & $30[\mathrm{~N} \cdot \mathrm{s} / \mathrm{m}]$ \\
\cline { 2 - 4 } & Right Hip & $80[\mathrm{Nm} / \mathrm{rad}]$ & $5[\mathrm{Nm} \cdot \mathrm{s} / \mathrm{rad}]$ \\
\cline { 2 - 4 } & Right Knee & $350[\mathrm{~N} / \mathrm{m}]$ & $30[\mathrm{~N} \cdot \mathrm{s} / \mathrm{m}]$ \\
\hline
\end{tabular}

actual hand placement used by trainers, who apply pressures to the flexor tendons during flexion and extensor tendons during extension to facilitate proper muscle activation.

\section{B. Force Control Algorithm}

We model the compressive airflow dynamics for each chamber of the pneumatic cylinders, and achieve force tracking by canceling the nonlinear terms using feedback. This algorithm employs two servovalves per cylinder to enable independent control of pressure on each side of the piston, although theoretically only one servovalve is required to control the net force output of a cylinder. The advantage of having extra control of individual pressures is that we can prevent undesirable pressure buildup, which causes increased seal friction in the actuator and a reduced passive compliance, leading to a poorer performance while in backdrive mode. We refer the reader to [18], [21], and [22] for more details.

\section{Position Control Algorithm}

To achieve position control, we adopt the hierarchical control strategy that was successfully applied to pneumatic actuators by McDonell [22]. The force-tracking controller acts as the inner loop, and the position controller as the outer loop.

We designed a simple proportional-plus-derivative (PD) control law in the task space coordinates shown in Fig. 1

$$
\tau_{d}=G_{P}\left(x-x_{d}\right)+G_{D}\left(\dot{x}-\dot{x}_{d}\right)
$$

where $x$ and $x_{d}$ are the actual and desired position of the attachment belt, respectively. The two gain matrices, $G_{P}$ and $G_{D}$, are diagonal and adjusted for each component of the nine DOF separately (Table I). We currently do not use off-diagonal elements (coupling of DOF), a feed-forward term, or a computed-torque type term, although these approaches are possible. We apply a constant upward force in order to partially compensate for the weight of the device (typically $40-80 \mathrm{~N}$ ). The desired task-space forces, $\tau_{d}$, are decomposed into desired cylinder forces, and passed on to the force tracking controller, which works on each cylinder.

\section{Teach-and-Replay Algorithm}

We would like to simulate a common situation in step training where no or minimum assistance is given by trainers as long as the patient is able to sustain stable stepping motion by himself, but more assistance is provided "as needed" if the stepping pattern begins to degrade or collapse. The teach-and-replay control method is our approach to achieving this using PAM and POGO. First of all, we record the pelvic and the leg trajectory during stepping with the device attached to the individual, with the help of human trainers as necessary. During this "teaching" period, the device essentially acts as a motion-capture device, recording the trajectory as it tries to minimize resistance by controlling the net actuator forces to be zero (backdrive-mode). Once the step pattern is recorded this way, we compute a mean trajectory pattern by identifying step cycles in the recorded data, normalizing the time scale, and taking an average over them. Then, we replay the repeating sequence of the computed mean trajectory using the hierarchical controller described above. Note that the whole purpose of teach-and-replay scheme is to produce proper force commands in the right direction at the right time. We do not pursue precise position tracking.

As described in the introduction, we found out through preliminary experiments with unimpaired individuals that it was very difficult to maintain stable stepping when the device was replaying at the constant (mean) speed, even if the trajectory was just sampled from the same person. So, we developed an algorithm to adjust the speed of replay in real-time using the step timing information obtained from footswitches [19]. Namely, the replay speed was adjusted proportionally to the difference of the reference timer speed and the actual speed as defined by the inverse of the elapsed time between consecutive rising edges of footswitch signal (i.e., step period), and additionally, a small constant was added to the replay speed when the measured rising edge was leading the corresponding point of the replay timer, or subtracted when lagging. This algorithm solved the problem of keeping the robot synchronized to the ever-fluctuating stepping of the individual, enabling the device to "catch up" or "pause" as the individual inevitably stepped quicker or slower. However, this algorithm was based on threshold detection on a signal from footswitches, which sometimes can be noisy and unreliable. As a result, we occasionally observed glitches in the behavior of the device when the footswitch signal was erratic due to a change in the foot landing angle or other motion. In order to address this problem, we developed a new algorithm that achieves smoother and more robust synchronization performance without need for footswitches.

\section{E. Synchronization Algorithm}

The synchronization algorithm first estimates the actual gait timing based on the measured position and velocity signals from all nine DOF of PAM and POGO at time $t$, represented by $x(t)$, which is a 18 dimensional vector. We have a gait pattern (reference trajectory), $x_{d}(T)$, which is defined by the mean position and velocity trajectory as a function of normalized step period $T=\left[\begin{array}{ll}0 & 1\end{array}\right]$, where 0 corresponds to the beginning of step cycle and 1 to the end of step cycle. We define the "beginning" of a step by the minimum of the lateral position signal, that is, when the pelvis reached the leftmost position during the step, although it can be any other point in the step period as long as it is consistent. The state vector is parameterized by the ratio to the amplitude of the reference trajectory after subtracting an offset 


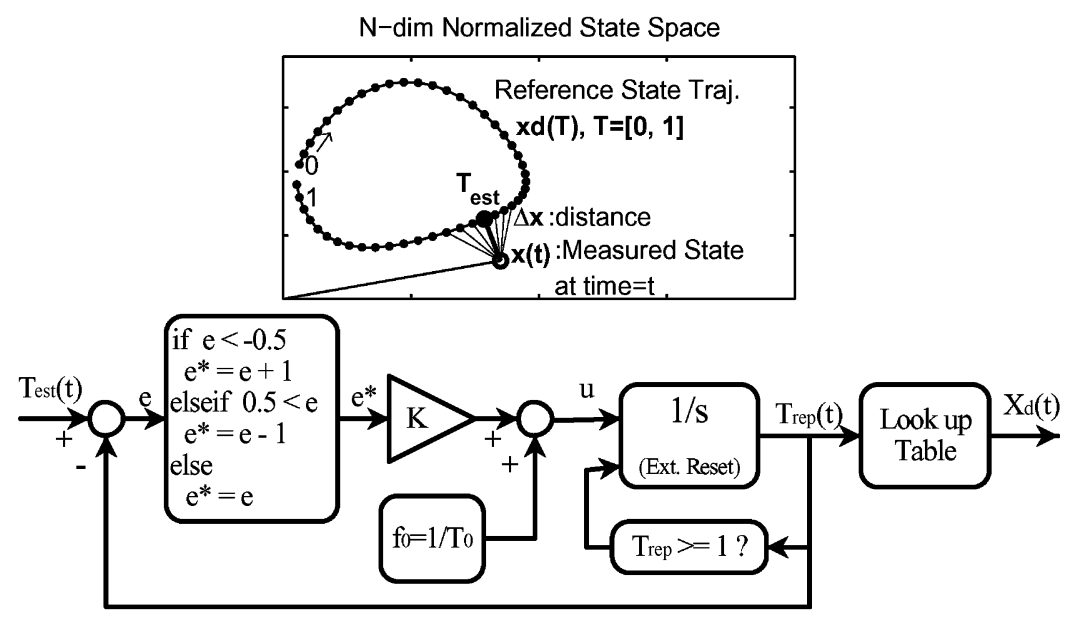

Fig. 2. Top: conceptual diagram of gait phase estimate by state comparison. Bottom: synchronization algorithm diagram.

(midrange of the reference trajectory), so that it is independent of the units used.

We obtain an estimate of the gait phase (timing) by computing the weighted vector norm of the difference between the measured state vector and all the points on the reference (mean) trajectory, and identifying the point with the least norm (Fig. 2). Namely, at time $t$, we compute

$$
\Delta x(T)=W\left\{x(t)-x_{d}(T)\right\} \quad \text { for } T=[0 \quad 1]
$$

where $W$ is the weight matrix, to find the minimum of $\operatorname{norm}(\Delta x)$ over $T$. We call the point that gives the minimum the estimate of gait phase, $T_{\text {est }}(t)$. Realistically, there will always be some amount of state error (i.e., norm $(\Delta x)$ will not be zero numerically). Conversely, the state error may get small for a moment even when the phase differs considerably. However, our assumption is that the error, when computed across 18 dimensions (positions and velocities) over time, should offer a reasonable indicator. This algorithm is actually more robust than the previous footswitch-based algorithm because it uses multiple sensor signals instead of one footswitch signal. We can optionally adjust relative influence of each of the 18 signals upon the outcome by the weight matrix $W$. For example, we could emphasize the importance of lateral position and rotation, while underemphasizing forward position, which can normally drift back and forth on the treadmill. In the experiments described in this paper, we set the weight to be uniform across all dimensions.

In our Matlab Simulink program, the repeating sequence of the desired pelvic trajectory is generated by accessing and interpolating the mean trajectory data stored in a lookup table. An integrator, with lower and upper saturation limits set to 0 and 1 , implements a variable-speed timer that feeds a normalized time index into the lookup table. The timer speed is thus governed by the input to the integrator. If the input to the integrator is constant at $1 / T_{0}$, where $T_{0}$ is the period of the original mean trajectory, the timer advances at "normal" speed. Once the timer saturates at 1 , a trigger resets the integrator to 0 , like flipping a sandglass. Taking advantage of the gait phase estimation, which we can obtain at any time during gait, we adjust the input to the integrator according to the feedback law described below. We define the normalized timing error at time $t$ as

$$
e(t)=T_{\text {est }}(t)-T_{\text {rep }}(t)
$$

where $T_{\mathrm{rep}}(t)=\left[\begin{array}{ll}0 & 1\end{array}\right]$ is the normalized replay time. Here, $e=$ -0.25 means the replay timer is leading the estimated actual gait phase by $25 \%$, and $e=+0.25$ means replay is lagging by $25 \%$. Now, we adjust the input to the integrator, $u$, so that $T_{\text {rep }}$ approaches $T_{\text {est }}$

$$
\begin{array}{ll}
\text { if } \quad-1 \leq e<-0.5 & \text { then } u=f_{0}+K(e+1) \\
\text { if } \quad-0.5 \leq e \leq 0.5 & \text { then } u=f_{0}+K(e) \\
\text { if } \quad 0.5<e \leq 1 & \text { then } u=f_{0}+K(e-1)
\end{array}
$$

where $K$ is the feedback gain and $f_{0}=1 / T_{0}$ corresponds to the normal replay speed. After an initial trial-and-error experiment with unimpaired subjects, $K=2.5$ was chosen for the experiments described below. Simply put, the replay timer is accelerated or decelerated in proportion to the difference of the current timer state and the estimated gait phase. This works in a continuous manner, as opposed to the discrete (once a step cycle) operation of the previous footswitch-based algorithm. Equations (4) and (6) are incorporated because when replay is leading or lagging by more than $50 \%$, it makes more sense to "skip" a cycle. Additionally, we apply saturation to the integrator input, $u$, so that the timer does not move too fast or go "backwards." The algorithm is summarized in Fig. 2.

\section{EXPERIMENTAL DESIGN}

We conducted experiments in order to verify the basic design of PAM and POGO and the controller. We assessed the accessibility of the system for a wheel chair, the adjustability of attachment braces, and the utility of pneumatics in a training environment. We also examined the feasibility of the robotic control schemes described above, namely, teach-and-replay and the real-time synchronization algorithm.

\section{A. Research Population}

Three unimpaired (UNI) and five chronic spinal cord injured (SCI) individuals participated in the experiment (Table II). A 
TABLE II

RESEARCH POPULATION

\begin{tabular}{l|l|l|l|l|l}
\hline ID & Sex & Age & BW & BWS & TM Speed \\
\hline \hline UNI1 & M & 39 & $802[\mathrm{~N}]$ & $50 \%$ & $0.89[\mathrm{~m} / \mathrm{s}]$ \\
\hline UNI2 & F & 30 & $652[\mathrm{~N}]$ & $50 \%$ & $0.89[\mathrm{~m} / \mathrm{s}]$ \\
\hline UNI3 & F & 27 & $553[\mathrm{~N}]$ & $50 \%$ & $0.89[\mathrm{~m} / \mathrm{s}]$ \\
\hline \hline SCI1 & M & 23 & $604[\mathrm{~N}]$ & $65 \%$ & $0.89[\mathrm{~m} / \mathrm{s}]$ \\
\hline SCI2 & M & 32 & $773[\mathrm{~N}]$ & $50 \%$ & $0.89[\mathrm{~m} / \mathrm{s}]$ \\
\hline SCI3 & F & 54 & $460[\mathrm{~N}]$ & $29 \%$ & $0.89[\mathrm{~m} / \mathrm{s}]$ \\
\hline SCI4 & F & 51 & $561[\mathrm{~N}]$ & $20 \%$ & $0.80[\mathrm{~m} / \mathrm{s}]$ \\
\hline SCI5 & M & 61 & $784[\mathrm{~N}]$ & $23 \%$ & $0.89[\mathrm{~m} / \mathrm{s}]$ \\
\hline
\end{tabular}

TABLE III

INJURY LEVEL

\begin{tabular}{l|l|l|l|l}
\hline ID & $\begin{array}{l}\text { Lesion } \\
\text { Level }\end{array}$ & $\begin{array}{l}\text { Years } \\
\text { Post } \\
\text { Injury }\end{array}$ & ASIA & Motor Score R/L \\
\hline \hline SCI1 & C5-6 & 4 & C & $9 / 9(0) /(3)$ \\
\hline SCI2 & C5-6 & 15 & D & $37 / 35(21) /(17)$ \\
\hline SCI3 & C6 & 10 & D & $40 / 37(\mathrm{n} / \mathrm{a}) /(\mathrm{n} / \mathrm{a})$ \\
\hline SCI4 & C5 & 7 & D & $41 / 46(21) /(22)$ \\
\hline SCI5 & C6-7 & 6 & D & $47 / 35(24) /(19)$ \\
\hline
\end{tabular}

$\mathrm{n} / \mathrm{a}$ refers to not available. Lesion Level: $\mathrm{C}=$ Cervical. ASIA C: Motor function is preserved below the neurological level, and more than half of key muscles below the neurological level have a muscle grade less than 3.

ASIA D: Motor function is preserved below the neurological level, and at least half of key muscles below the neurological level have a muscle grade greater than or equal to 3 .

(parentheses) in motor score indicate the lower limb component of the total motor score.

clinician assessed the level and extent of SCI according to the American Spinal Injury Association (ASIA) impairment scale [23]. All individuals had previously received locomotor training using BWS on a treadmill. None of the SCI individuals were taking antispasticity medication at the time of the experiment. Injury level, years postinjury, and ASIA impairment scale and motor scores for each individual are summarized in Table III. The University of California at Irvine and the Los Angeles Institutional Review Boards approved all experiments, and each individual signed an informed consent form prior to participating in the study.

\section{B. Protocol}

All individuals wore a harness and were suspended by the overhead BWS system. For all SCI individuals, the speed of the treadmill and level of body weight support were adjusted per individual by an experienced physical trainer based on the quality of stepping and safety of the person and were held constant throughout the experiments (Table II). For all the unimpaired individuals, BWS was chosen at $50 \%$ of their body weight.

Experienced trainers provided manual assistance at the hips, knees and ankles for the SCI individuals as necessary during stepping. The trainers used a hand placement over the patella tendon to assist in knee extension during the stance phase, and at the popliteal crease for hip and knee flexion during swing.
TABLE IV

EXPERIMENTAL PROTOCOL

\begin{tabular}{|c|c|c|}
\hline (A) & No-Robot & Warm-up, Find base EMG. \\
\hline \multirow[t]{2}{*}{ (B) } & PAM Backdrive & Motion-capturing \\
\hline & & Compute the mean pelvic trajectory \\
\hline \multirow[t]{2}{*}{ (C) } & PAM Replay & Replay the mean pelvic trajectory \\
\hline & with Synch & with synchronization. \\
\hline \multirow[t]{2}{*}{ (D) } & PAM Replay & Replay the mean pelvic trajectory \\
\hline & without Synch & at constant speed. \\
\hline \multirow[t]{2}{*}{ (E) } & PAM Synch & Motion-capturing \\
\hline & POGO Backdrive & Compute the mean leg trajectory. \\
\hline \multirow[t]{3}{*}{ (F) } & PAM Synch & Replay the mean pelvic and leg \\
\hline & POGO Synch & trajectory with synchronization. \\
\hline & & Manually adjust Scale/Offset. \\
\hline
\end{tabular}

The trainers used a hand placement just proximal to the ankle to assist proper foot placement and foot clearance at liftoff. A trainer positioned behind the individual aided in hip stabilization and weight shifting during stepping as needed.

For all individuals, we recorded electromyographic (EMG) activity and footswitch signal at $1 \mathrm{kHz}$ using a 24-channel hard-wired analog-to-digital board and a custom-written Labview software acquisition program (National Instruments, Austin, TX). EMG activity was measured bilaterally from the soleus (SL), medial gastrocnemius (MG), tibialis anterior (TA), medial hamstrings (MH), vastus lateralis (VL), and rectus femoris (RF) using bipolar surface electrodes [24], then sampled and ac-coupled into a differential amplifier (Konisberg Instruments, Pasadena, CA).

In order to examine the effect of the robot attachment on the individuals, and to compare the effectiveness of different control methods, we examined six experimental conditions (Table IV).

In condition (A), the individual walked with no robot attached as a warm-up while EMG and footswitch signals were recorded. The trainers assisted the individual in stepping as needed. Then, in condition (B), PAM was attached to the individual. POGO was not attached at this point. After the individual walked with PAM for a minute or two to get used to it, we recorded the pelvic trajectory, EMG, and footswitch signals for about $30 \mathrm{~s}$ in the backdrive-mode, that is, PAM was yielding to the forces applied to it (zero force control), allowing naturalistic step kinematics with only light damping. We asked the unimpaired individuals to somewhat exaggerate their normal pelvic motion since the device tended to impede it slightly even in zero force control mode. With the SCI individuals, we asked the trainers to provide assistance so that there was a stable and normal-as-possible motion of the pelvis and legs, which is what the trainers typically do during training. We then computed the mean trajectory from the kinematics data just captured, which we used as the reference trajectory for the following two conditions.

In condition $(\mathrm{C})$, the computed mean trajectory was replayed with the synchronization algorithm. In condition (D), the mean pelvic trajectory was replayed at the constant speed without synchronization. In our previous experiments with unimpaired individuals, this condition (D) caused a kind of "beat" phenomenon, in which the individual goes in and out of synchronization with the robot typically at a frequency of $0.05-0.1 \mathrm{~Hz}$. The 


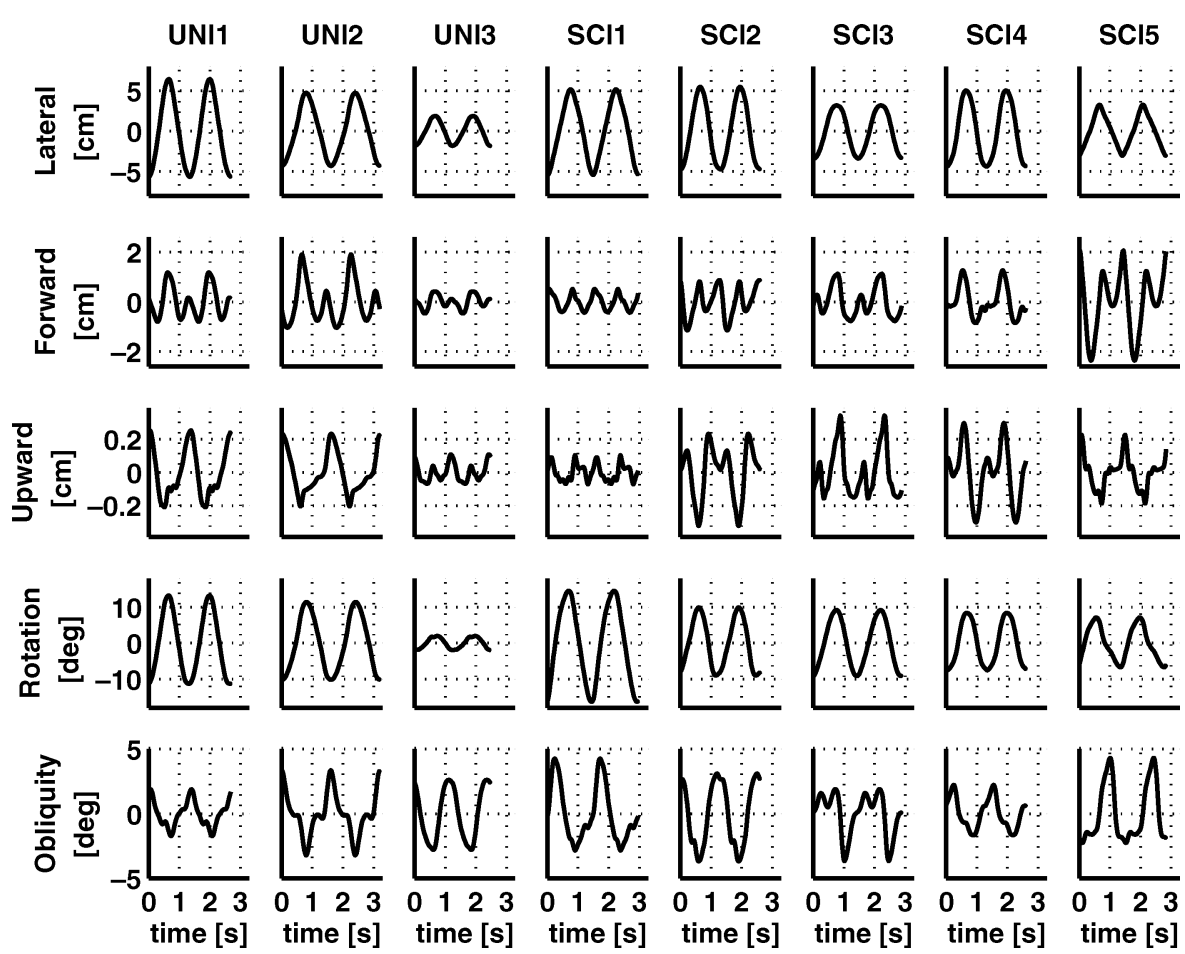

Fig. 3. Two cycles of the mean trajectories. Each column represents the mean pelvic trajectory from an unimpaired (UNI) or spinal cord injured (SCI) individual. The rows represent five axes of PAM. Each individual exhibited a unique pelvic trajectory. The scale is shifted such that the trajectory has zero mean.

order of the two conditions, (C) and (D), was randomized. Also during these conditions, the trainer at the pelvis assisted only if the pelvic motion became too erratic. For the trainers at the legs, we asked them to provide as little assistance as necessary to maintain a safe and stable gait, and let go of the individual if possible.

In condition (E), we attached POGO in addition to PAM. POGO was set in the backdrive-mode, while PAM synchronously replayed the reference (mean) trajectory just as it did in condition (C), thus acting in place of the trainer at the pelvis. The trainers assisted at the legs as needed. Once a consistent step pattern was established, we recorded the trajectory of PAM and POGO for about $30 \mathrm{~s}$. From the recorded data, we computed the mean trajectory for both PAM and POGO, which was then used as the reference trajectory in the following condition (F). In condition (F), PAM and POGO actively assisted at the pelvis and legs with synchronization. In an attempt to provide the trainers at the legs with more power assistance, we manually adjusted the amplitude and the offset of the reference trajectory. Specifically, we increased the amplitude of the desired (reference) trajectory to assist more in the leg swing, and additionally applied negative offset to the desired knee cylinder trajectory so as to prevent the knee buckling during stance. The protocol is summarized in Table IV.

\section{RESULTS}

\section{A. Kinematics}

Each individual exhibited a unique pelvic trajectory as measured by the device (Fig. 3). The ranges of motion were larger in lateral translation and pelvic rotation than forward, upward, or in obliquity. Pelvic motion in the forward direction repeated a pattern at double the frequency of lateral and pelvic rotation.

The synchronization algorithm made the kinematics more consistent. Fig. 4 shows a sample of lateral pelvic trajectory of SCI1 (ASIA: C) and SCI2 (ASIA: D) for the three conditions: (B: Backdrive mode with human trainer assisting), (C: robot assisting with synchronization), and (D: robot assisting without synchronization). The top row indicates the trajectory for condition (B), where PAM was operated in the backdrive-mode, from which we computed the mean trajectory. Although the waveform is slightly different from one period to another, we see a pattern that repeats fairly consistently thanks to manual assistance by the trainers. The next two rows show the desired and the actual trajectories when PAM was replaying with synchronization (middle row) and without synchronization (bottom row). The "beat" phenomenon is evident in the bottom row, where the actual trajectory lacks consistency, even though the desired trajectory was the exact repetition of the mean trajectory. The resulting actual trajectory shows a gradual decrease in the amplitude ( $0-10 \mathrm{~s})$, then an increase, accompanied by deformation of the waveform. In the middle row, however, when PAM synchronized itself to the timing of the actual steps, the resulting trajectory maintains a consistent pattern. This is a clear contrast from the "beat" phenomenon. For SCI2, we observed a consistent gain in the amplitude, which had an effect of encouraging weight shifting. With synchronization, the trainers were able to let go of the pelvis and were more comfortable guiding the legs.

Fig. 5 summarizes the mean of the amplitude of lateral pelvic trajectory for each individual with the standard deviation bar. 

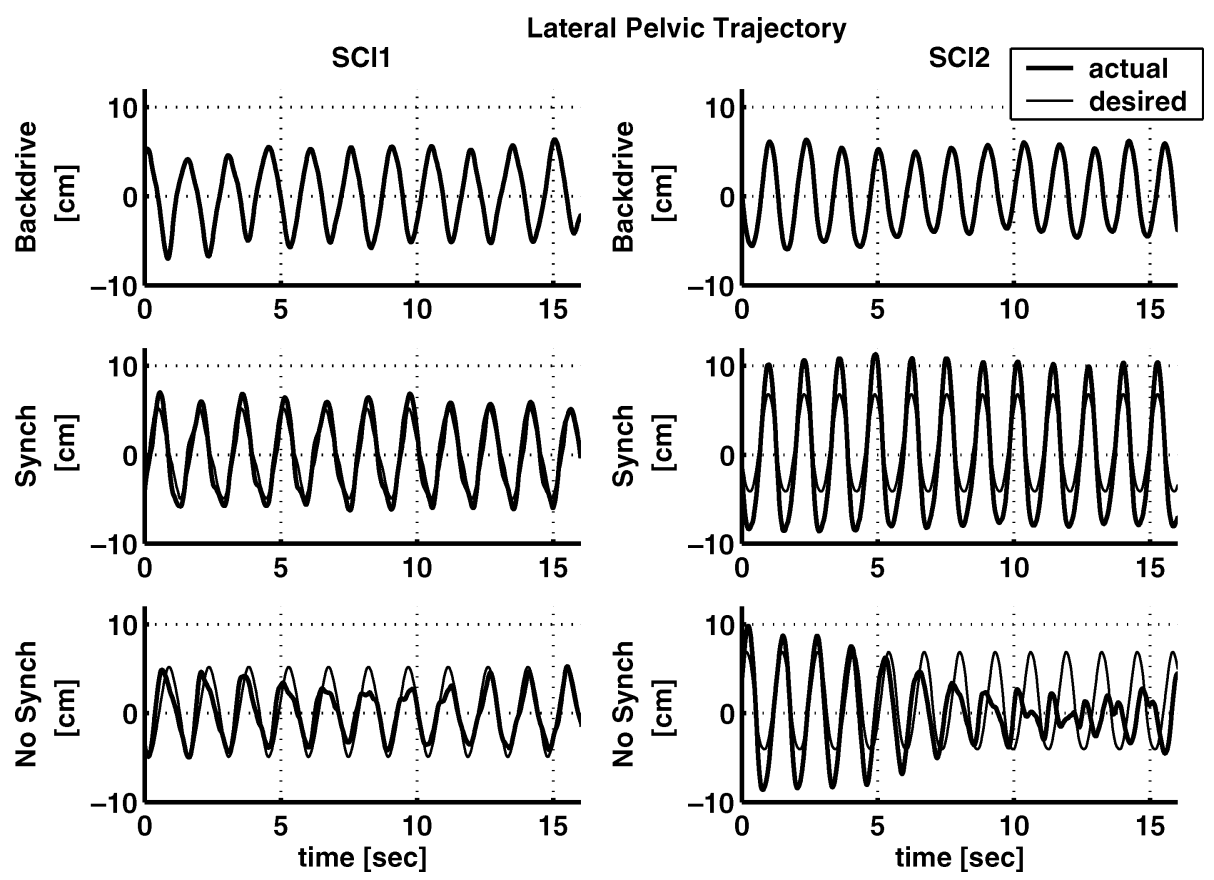

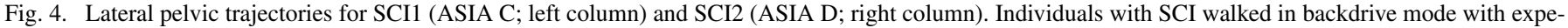

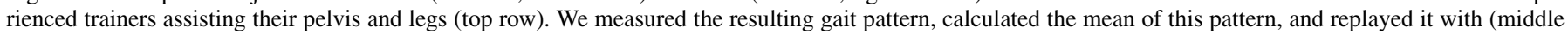

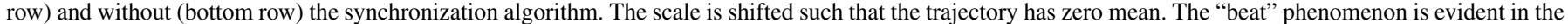
bottom row. A resonance-like phenomenon is observed for SCI2 with synchronization.

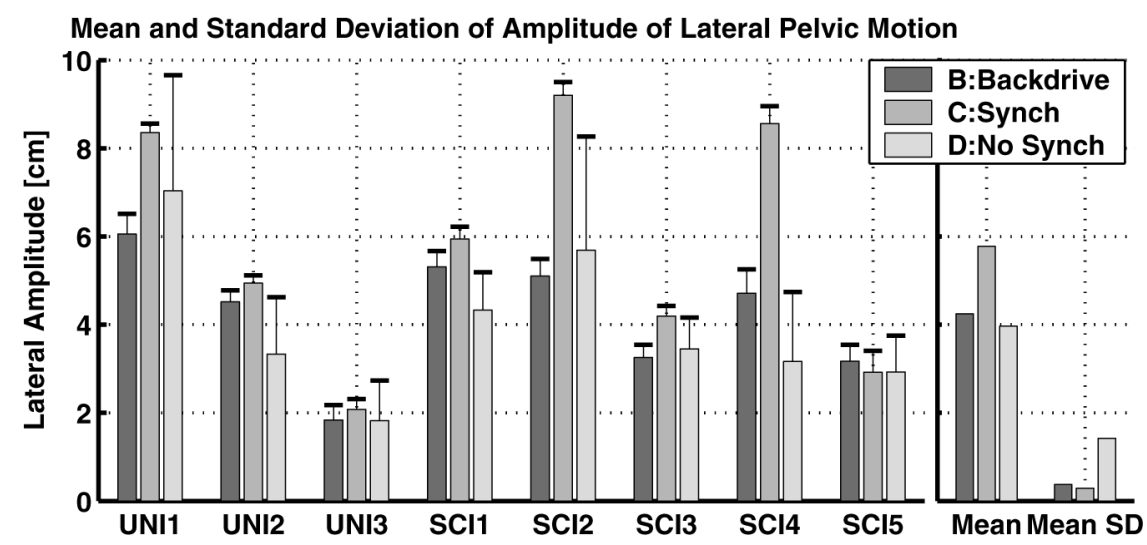

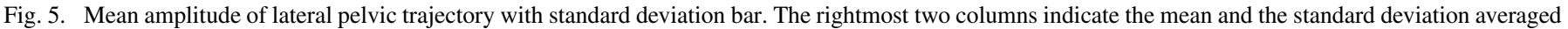
across all individuals.

The rightmost two columns indicate the mean across all individuals of the mean amplitude and the standard deviation. Generally, the mean amplitude increased in condition (C: Synch), in a sort of resonance-like behavior, as compared to condition (B: Backdrive) or condition (D: No-Synch). The standard deviation of the amplitude for each individual, averaged across individuals, was significantly larger for condition (D: No-Synch) compared to condition (B: Backdrive) and (C: Synch) ( $t$ test, $p<0.006$ ), corresponding to the "beat" phenomenon.

We computed the phase lag of lateral pelvic motion relative to actual step of the foot (Fig. 6) to see the effect of synchronization more closely. We identified timing of the local maxima of the lateral trajectory (when it hits the rightmost position in each cycle) and the rising edges (loading) of the right footswitch signal, and normalized the time difference by the mean step period. A phase lag of, for example, $25 \%$ indicates that the pelvis reached the rightmost position of the cycle following the right foot landing by $25 \%$ of the mean period, and a negative lag means that the pelvis preceded the foot landing (lead). Note that the synchronization algorithm is not designed to drive the lag to zero; the phase relationship between the pelvis and the foot should ideally stay constant. Overall, the phase lag stayed fairly constant under conditions (B: Backdrive) and (C: Synch), meaning the temporal relationship of the pelvic motion and the foot landing was consistent. However, it varied greatly under condition (D: No-Synch). This indicates that the timing relationship of the pelvis and the foot kept changing step after step, causing the participants to experience seemingly random forces that pushed their pelvis, impeding the consistency of step pattern. Many times the phase lag grew larger and larger, suggesting that the individual took earlier, or shorter, steps repeatedly. For UNI1, the lag grew quickly over $100 \%$, meaning his 

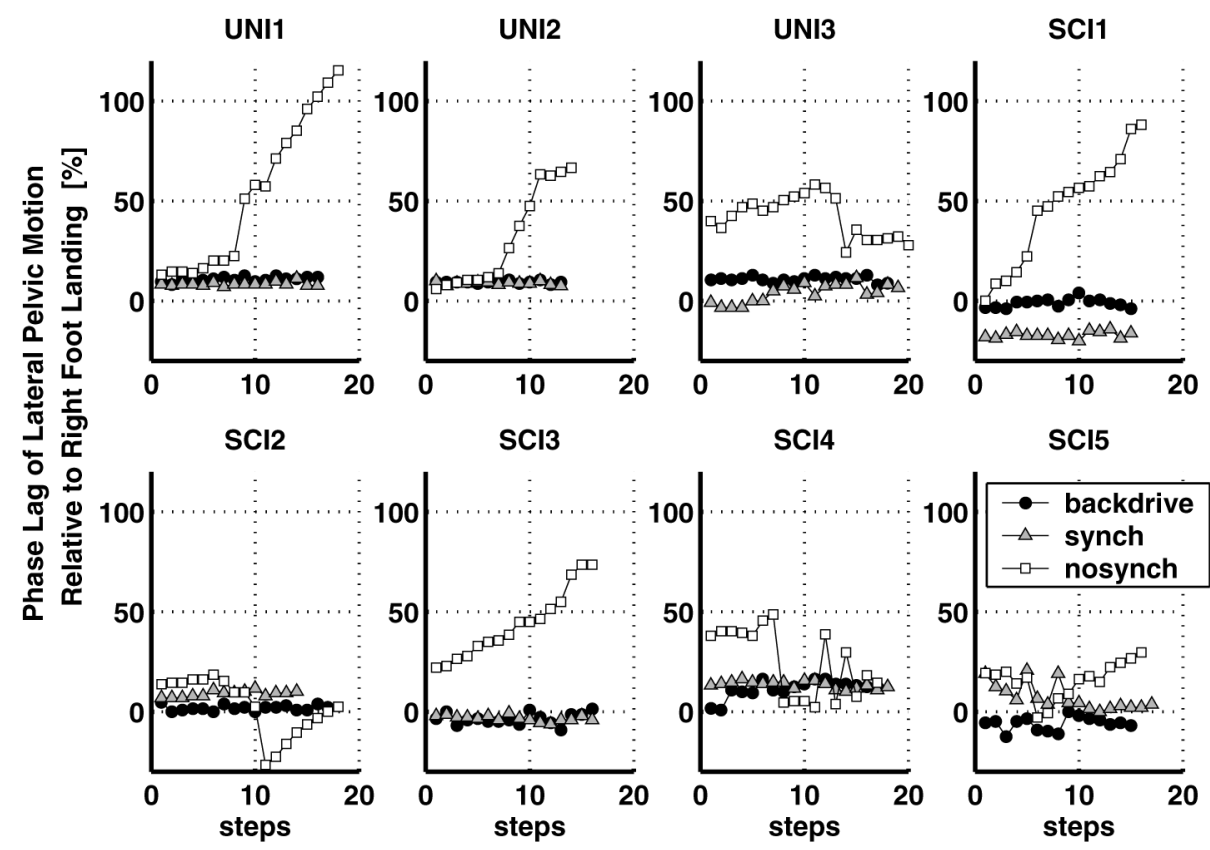

Fig. 6. Phase lag of lateral pelvic motion relative to right foot, shown for each individual. Positive phase lag means that the pelvis hit the rightmost position of the cycle after the right foot landing. Large variation is observed for the No-Synch case, indicating inconsistency of timing relationship of the pelvis and right foot.

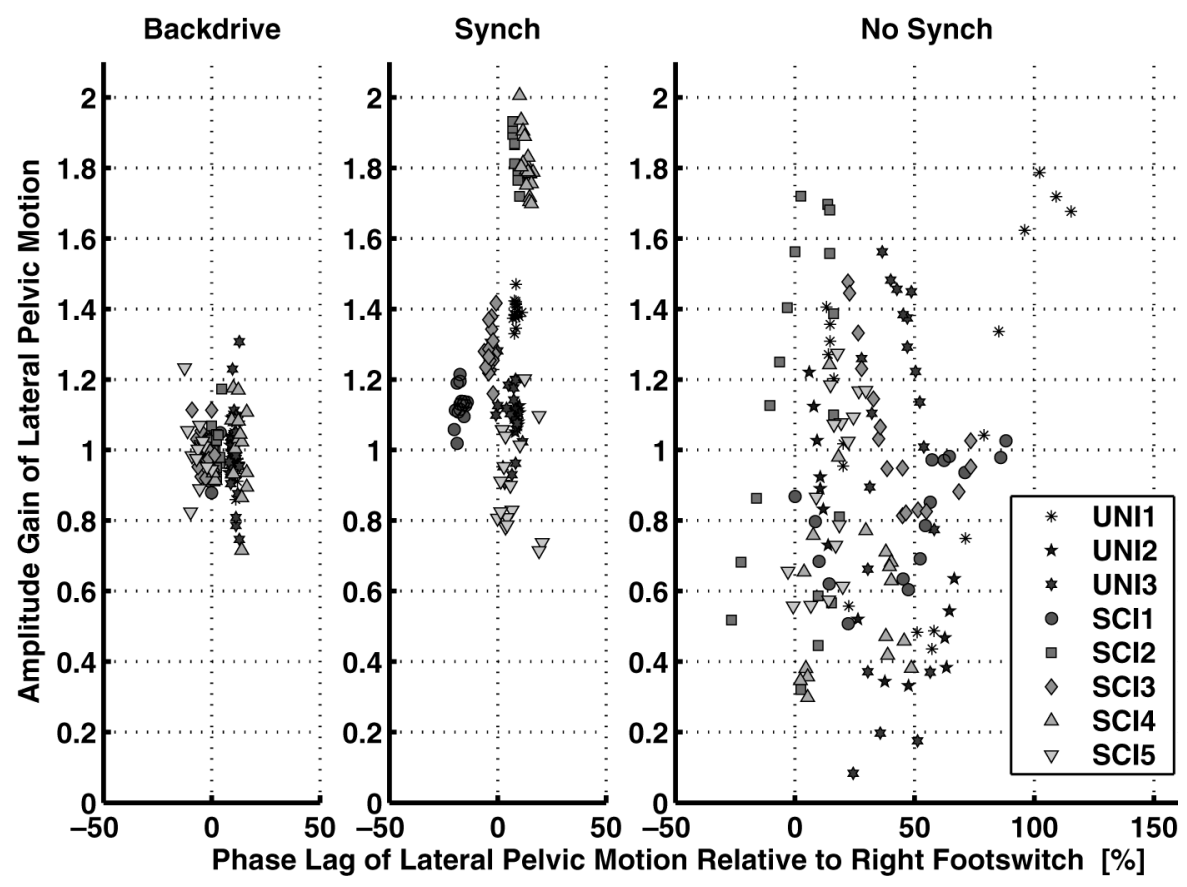

Fig. 7. Lateral amplitude gain versus Phase lag. Left: We set the mean of this data as the base amplitude, where PAM was operated in the backdrive-mode with trainers assisting. Middle: When PAM assisted with the synchronization algorithm, the phase lag remained fairly constant for each individual. Right: Without the synchronization algorithm, the amplitude varied greatly as the phase lag kept changing.

feet made one more step than his pelvis did within the 25 -s period. For SCI1, the most severely impaired patient (ASIA: C), we can observe a sort of steady-state phase lead with synchronization. This implies that PAM was literally leading the patient, actively driving the pelvis to encourage foot motion consistently.

Fig. 7 shows the amplitude gain of the lateral pelvic motion versus the phase lag. For each research participant, we set the mean amplitude from condition (B: Backdrive) to be the base amplitude. Therefore, for condition (B: Backdrive) on the leftmost column, the data points are distributed around unity gain by definition. For condition (C: Synch), we see increase an in amplitude for most individuals, and the phase lag remained fairly constant. The overall data points are tightly clustered within each individual. However, for condition (D: No-Synch) in the rightmost column, we see a different distribution of data points. The amplitude varied greatly as the phase lag kept changing. It appears that in condition (C: Synch) 


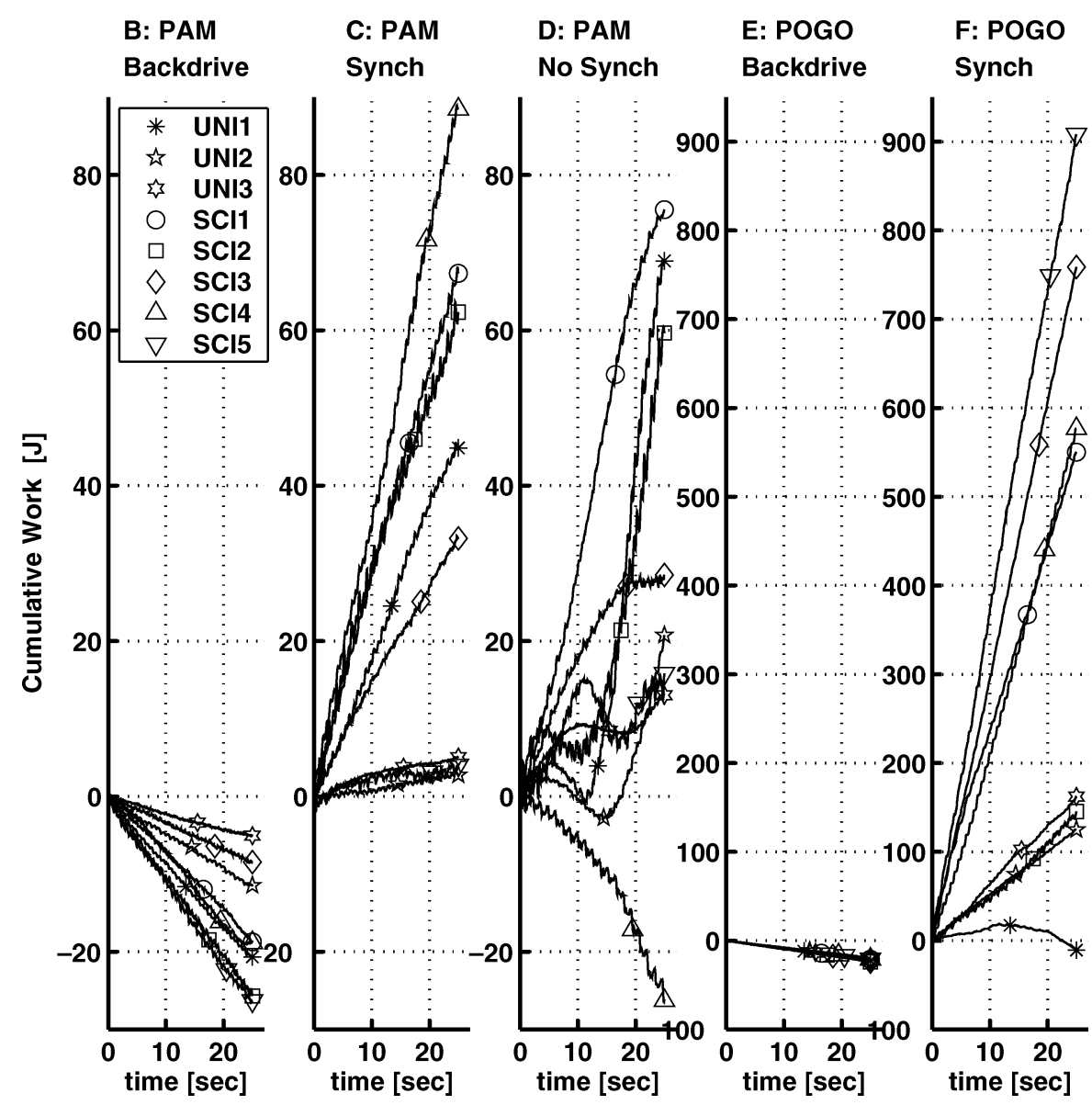

Fig. 8. Cumulative work done by PAM (B, C and D) and work done by POGO (E and F). Negative slopes indicate dissipation of energy (B and E). Steady positive slopes indicate consistent power delivery (C and F). The ebb and flow of the slopes suggest inconsistent power delivery (D). With manual adjustments of the amplitude and offset of the desired trajectory, POGO generated greater work than PAM did by a factor of ten.

the synchronization algorithm kept the phase around the peak band, causing a sort of resonance, whereas in condition (D: No-Synch) transition between the resonance and interference was observed.

\section{B. Power Analysis}

The kinematic patterns described above are the result of complex dynamics, which we do not know in detail since we do not have an exact model or knowledge of all the forces in the system. However, we can get an indication of the general level of robotic assistance by analyzing the total mechanical power generated by the device. When the generated power is positive, we can consider that the device is in general driving itself and what is attached to it, namely the individual's pelvis and possibly the hands of the trainers. When it is negative, the device is generally being driven by the individual and/or the trainers. We estimated the instantaneous power by computing the product of force and velocity for each cylinder, and summing over all cylinders, where the forces were calculated from measured pressures and the velocity from taking numerical derivative of measured cylinder lengths. The resulting value is therefore an estimate and contains some noise. To see the general trend of flow of power more clearly, we then computed the accumulation of power, that is, mechanical work done, or energy generated by the device.
More specifically, we computed the time integral of the instantaneous power.

The left three columns in Fig. 8 show the resulting total work by PAM as it accumulated over time. In condition (B: Backdrive) on the leftmost column, the steady negative slope indicates dissipation of energy due to the damping effect of the device. In condition (C: Synch), where PAM was actively replaying the reference (mean) trajectory while adjusting the timing, the steady positive trend of the slope indicates that the device was adding energy into the system consistently. We notice that more power, as indicated by steeper slope, was delivered to participants with SCI than the unimpaired individuals in general. In contrast, the ebb and flow of the slope in condition (D: No-Synch), where PAM was replaying at the fixed step period of the computed mean, suggest inconsistent power delivery. The direction of power flow changed every $10 \mathrm{~s}$ or so, and the effect was more disturbing than assistive. Indeed, the trainers at the legs mentioned that they constantly had to work harder to stabilize the whole stepping motion in condition (D: No-Synch) than in any other condition, although the trainer at the pelvis was comfortable letting go, as far as stability and safety are concerned, for all individuals under all conditions except for some cases of quick transition between in-synch and out-of-synch states in condition (D). 

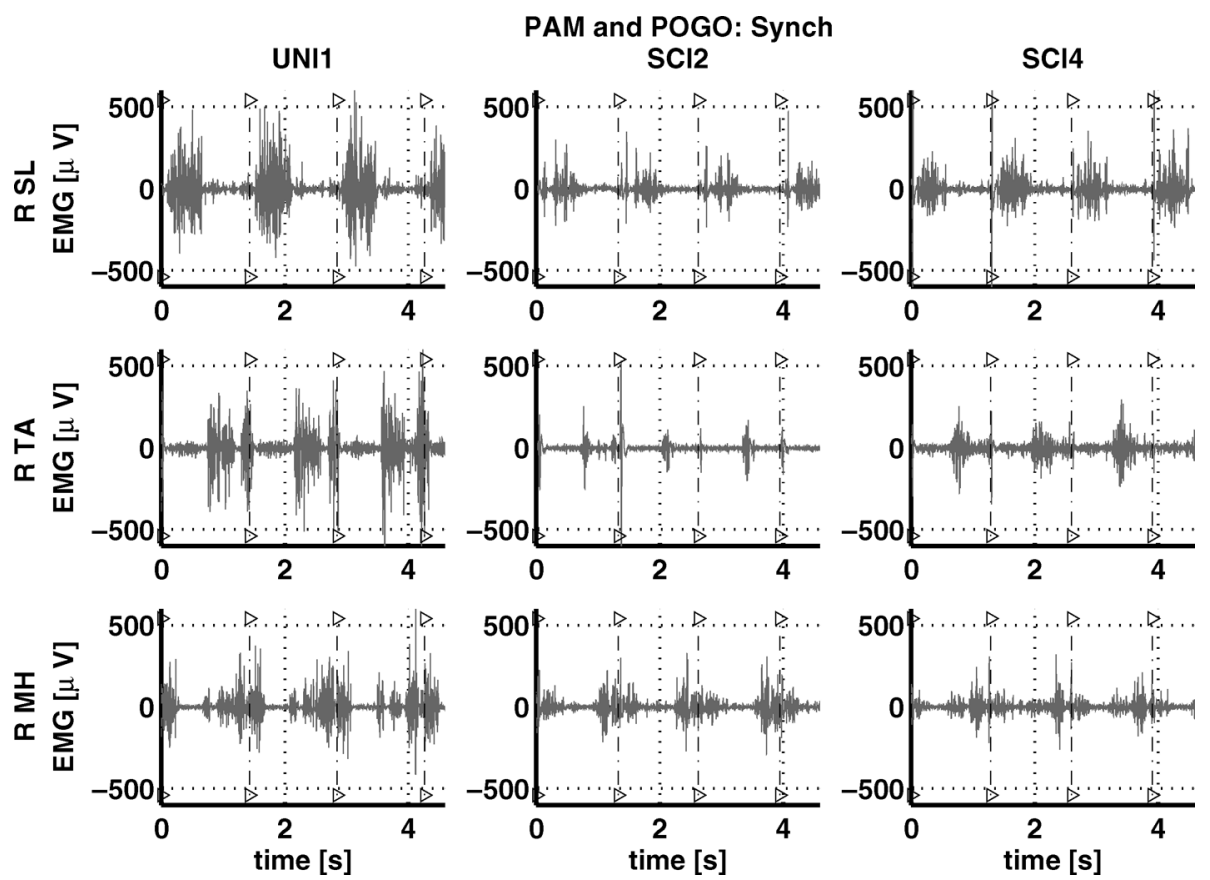

Fig. 9. Sample of raw EMG activity. Each column represents data from UNI1, SCI2 and SCI4, with each row representing muscle groups: Right SL, Right TA and Right MH. The vertical dashed lines with triangles at both ends indicate the timing of rising edges (loading) of right footswitch signal.

The right two columns in Fig. 8 show the total work done by POGO. Again, the condition (E: POGO Backdrive) resulted in dissipation of energy as indicated by small negative slope for all individuals, and the condition (F: POGO Synch) shows consistent power delivery. We note that the amplitude and offset of the desired trajectory for POGO had been manually adjusted in response to the trainers' request. As a result, we were able to provide more power assistance with SCI individuals. The amount of power generated by POGO was larger than that by PAM by a factor of ten. Among all conditions, the most preferred by trainers was condition (F: POGO Synch), where they could let go most comfortably with the least need for extra stabilization or excitation. An overall small negative trend is observed in the condition (F: POGO Synch) for UNI1, whereas all other participants show positive trends at varying degrees. This comes from the fact that UNI1, who is tall and healthy, was able to walk by himself, providing the power to move the legs. The slight positive trend in the first $15 \mathrm{~s}$ corresponds to the period when he was obtaining the feel of the robotic assistance, and the following negative trend implies that he had found a comfortable pace without relying on the device.

\section{C. $E M G$}

We measured EMG from several leg muscle groups as an indication of amount and timing of muscle activation. Fig. 9 shows a sample of EMG signal for select research participants and muscle groups recorded under condition (F), where PAM and POGO were actively assisting with synchronization. The vertical dashed lines with triangles at both ends indicate the rising edges (loading) of right footswitch signal. We filtered the raw EMG signal by taking the root mean square of a moving window of $100 \mathrm{~ms}$, sectioned the RMS-filtered EMG signal at the rising edges of right footswitch signal, normalized the time scale to the mean step period, and took the mean over 15-20 cycles from the 25-s period. Fig. 10 shows the resulting mean EMG for select individuals and muscles for conditions (A: No-Robot), (E: PAM Synch and POGO Backdrive) and (F: PAM and POGO Synch). We did not detect a coherent pattern or rule of EMG variation as a function of different control methods of PAM and POGO. The analysis is not conclusive, but it appears that EMG patterns are similar whether the assistance was given by trainers or the robot.

\section{DISCUSSION/CONCLUSION}

Our first objective for these initial experiments was to verify the feasibility of attaching and operating PAM and POGO in a typical training environment. The result was positive in that both unimpaired individuals and individuals with SCI tolerated the device well from the start without any previous exposure to it, although they were familiar with locomotor training using BWST. However, we spent a considerable amount of time, up to $30 \mathrm{~min}$ for some individuals, adjusting the attachment braces. This problem of securing physical interface between machine and human individual is an area that needs to be addressed in the next stage of development.

All research participants were able to walk without experiencing unnatural hard constraints. The inertia of the device was not large enough to hinder the research participants or the trainers, although the slight damping effect reduced the amplitude of the pelvic motion to some extent in the backdrive-mode. Nevertheless, the device successfully provided a motion-capturing function for our purpose. The captured pattern of pelvic motion was unique to each individual, disabled or unimpaired, suggesting that it is more desirable to tailor the desired trajectory for each individual than applying a certain trajectory taken from someone else. 

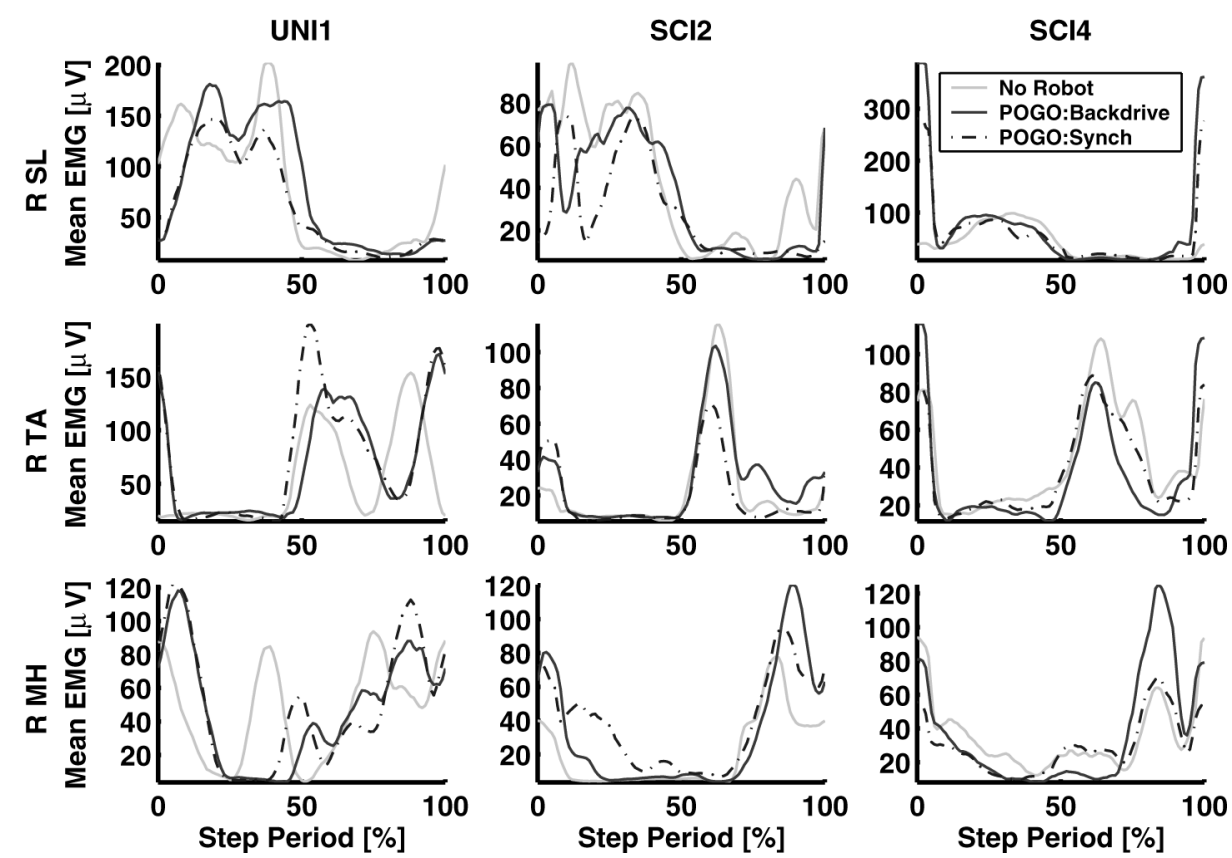

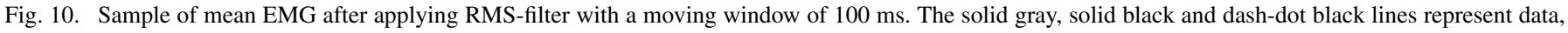

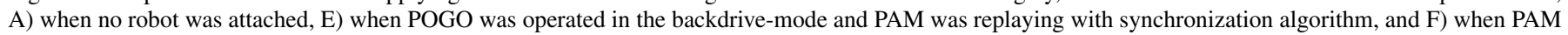
and POGO were assisting with synchronization, respectively.

Due to the compliant characteristics of the device, we encountered the problem of desynchronization (the "beat" phenomenon) during fixed-speed teach-and-replay with individuals with SCI as well as unimpaired individuals. In general, both unimpaired and SCI individuals showed a tendency to take shorter and quicker steps on average when they were attached to the device. The most likely explanation is that the device, when out of synchronization, applied "unexpected" forces to the individuals, and as a result, the individuals tried to gain stability against the robotic forces by attaining double-stance phase, which leads to shorter steps given a constant treadmill speed. A similar timing mismatch occurs and grows if the individual trips, and then tries to regain balance by making a few quicker steps to pick up the pace.

The synchronization algorithm eliminated the beats effectively, delivering consistent power assistance as manifested by the analysis of robotic power and work. The mean EMG patterns were generally similar across conditions, although a more rigorous analysis of EMG would require many more research participants. With POGO helping them, the trainers were able to let go of the knees and the ankles completely for a short duration of time for most individuals with SCI. With the most severely disabled individual (SCI1: ASIA C, wheelchair-bound), the trainers were still able to let go of the knees completely and only needed to apply light support to prevent toe drop. PAM with synchronization consistently worked well in place of the trainer behind the individual, scarcely requiring additional assistance from the trainer. The subjective impression of the physical therapists and trainers was that PAM satisfactorily assisted in pelvic motion. For POGO, the physical therapists' main criticism was related to the difficulty in appropriately adjusting the knee and ankle attachment braces.

The synchronization algorithm appeared to produce a sort of resonance, as evidenced by the larger amplitude of resulting lateral pelvic motion compared to the desired trajectory. This finding suggests that forces were being applied in such a direction at such a time as to pump energy into the system. Whether this is always desirable is uncertain. The trainers did not remark that this was undesirable, suggesting that they were more concerned with the overall timing of the gait pattern, which was appropriate. We can adjust the level of power delivery, if desired, by changing the controller gains and scaling the desired trajectory. We can also specify the assistance and nonassistance for each axis. For example, we can command a stiffer gain and larger desired trajectory in pelvic rotation to provide more assistance, while allowing free (backdrivable) lateral motion by applying low or zero gain. Moreover, by scaling the amplitude of the reference trajectory, the device can either approach the effect of a stabilizing spring-damper (by decreasing reference amplitude) or alternatively promote larger motion (by increasing amplitude). Therefore, the device can potentially make a transition between "stabilizer" and "promoter" as desired. We could also schedule the PD gains as a function of the gait phase. This would allow us to specify, for instance, more lateral support during swing phase and/or more rotational support at foot landing and so on.

With the synchronization algorithm, the teach-and-replay scheme used here offers a feasible approach to the goal of providing robotic assistance as needed. We note that the desynchronization problem will likely be a general problem for any compliant robot that is assisting in a naturalistic motion. The algorithm developed here solves this problem by comparing the current state (position and velocity) of the individual to the desired state (a known pattern), then accelerating or decelerating the timing of the replayed pattern to bring the two into correspondence. We speculate that this approach may provide a general solution for temporal, and consequently spatial, alignment of compliant assistance. 
Our objective was to design a robotic device that can provide assistance in gait training only "as needed." To that aim, we developed unique robotic devices: PAM and POGO. In contrast to existing devices, such as the MGT, the Haptic Walker and the Lokomat, PAM and POGO are inherently compliant and allow naturalistic motion during treadmill walking. Moreover, PAM and POGO are designed to manipulate both the pelvis and the legs, whereas most existing devices primarily focus on the legs only and treat the pelvic motion as a mere supplement. Some other devices focus on the torso and the pelvis, but not the legs. For instance, the string-man is a wire-driven robotic device with force (tension) control capability to provide a virtual safety envelope in which the patient can practice balancing or walking [25], which is arguably like a complex form of a BWS system.

Just as the development of BWS systems freed trainers from the exhausting work of keeping the patient upright and balanced against gravity, we aim to develop a robotic device that can relieve, at least partially, the trainers from the demanding physical labor of supporting and guiding the patient through the stepping motion, so they can work longer and more efficiently while focusing more on the quality of the training. Although we are still uncertain of the clinical efficacy of step training with PAM and POGO using BWST, we successfully conducted initial experiments with individuals with a spinal cord injury that demonstrated that the device has significant potential for relieving the trainers of strenuous work, for enhancing therapy, and for assessing recovery.

\section{ACKNOWLEDGMENT}

The authors would like to thank all the volunteers who participated in the research and the staff at UCLA including J. Beres-Jones, A. Budovitch, R. van den Brand, and C. Dy.

\section{REFERENCES}

[1] V. Dietz, G. Colombo, and L. Jensen, "Locomotor activity in spinal man," Lancet, vol. 344, pp. 1260-1263, 1994.

[2] M. Visintin, H. Barbeau, N. Korner-Bitensky, and N. Mayo, "A new approach to retrain gait in stroke patients through body weight support and treadmill stimulation," Stroke, vol. 29, pp. 1122-1128, 1998.

[3] S. Hesse, M. Konrad, and D. Uhlenbrock, "Treadmill walking with partial body weight support versus floor walking in hemiparetic subjects," Arch. Phys. Med. Rehabil., vol. 80, no. 4, pp. 421-427, Apr. 1999.

[4] A. Wernig, A. Nanassy, and S. Muller, "Laufband (treadmill) therapy in incomplete paraplegia and tetraplegia," J. Neurotrauma, vol. 16, no. 8, pp. 719-726, Aug. 1999.

[5] A. L. Behrman and S. J. Harkema, "Locomotor training after human spinal cord injury: A series of case studies," Phys. Therapy, vol. 80, pp. 688-700, 2000.

[6] V. R. Edgerton, R. D. de Leon, S. J. Harkema, J. A. Hodgson, N. London, D. J. Reinkensmeyer, R. R. Roy, R. J. Talmadge, N. J. Tillakaratne, W. Timoszyk, and A. Tobin, "Retraining the injured spinal cord," J. Physiol., vol. 533, no. 1, pp. 15-22, May 2001.

[7] J. A. Galvez, G. Kerdanyan, S. Maneekobkunwong, R. Weber, M. Scott, S. J. Harkema, and D. J. Reinkensmeyer, "Measuring human trainers' skill for the design of better robot control algorithms for gait training after spinal cord injury," in Proc. IEEE 9th Int. Conf. Rehabil. Robot., Chicago, IL, Jun./Jul. 2005, pp. 231-234.

[8] S. Hesse and D. Uhlenbrock, "A mechanized gait trainer for restoration of gait," J. Rehabil. Res. Development, vol. 37, no. 6, pp. 701-708, 2000.

[9] S. Jezernik, G. Colombo, T. Keller, H. Frueh, and M. Morari, "Robotic orthosis lokomat: A rehabilitation and research tool," Neuromodulation, vol. 6, no. 2, pp. 108-115, Apr. 2003.
[10] V. T. Inman, H. J. Ralston, F. Todd, and J. C. Lieberman, Human Walking. Baltimore, MD: Williams Wilkins, 1986, pp. 41-45.

[11] J. M. Hidler and A. E. Wall, "Alterations in muscle activation patterns during robotic-assisted walking," Clin. Biomechan., vol. 20, no. 2, pp. 184-193, 2005.

[12] H. I. Krebs, B. T. Volpe, M. L. Aisen, and N. Hogan, "Increasing productivity and quality of care: Robot-aided neuro-rehabilitation," J. Rehabil. Res. Development, vol. 37, no. 6, pp. 639-652, 2000.

[13] J. L. Emken, J. H. Wynne, S. J. Harkema, and D. J. Reinkensmeyer, “A robotic device for manipulating human stepping," IEEE Trans. Robot., vol. 22, no. 1, pp. 185-189, Feb. 2006

[14] S. Jezernik, G. Colombo, and M. Morari, "Automatic gait-pattern adaptation algorithms for rehabilitation with a 4-dof robotic orthosis," IEEE Trans. Robot. Autom., vol. 20, no. 3, pp. 574-582, Jun. 2004.

[15] R. Riener, L. Lunenburger, S. Jezernik, M. Anderschitz, G. Colombo, and V. Dietz, "Patient-cooperative strategies for robot-aided treadmill training: First experimental results," IEEE Trans. Neural Syst. Rehabil. Eng., vol. 13, no. 3, pp. 380-394, Sep. 2005

[16] H. Schmidt, D. Sorowka, S. Hesse, and R. Bernhardt, "Design of a robotic walking simulator for neurological rehabilitation," in Proc. IEEE/RSJ Int. Conf. Intelligent Robots Syst., Oct. 2002, vol. 2, pp. $1487-1492$

[17] H. Schmidt, S. Hesse, R. Bernhardt, and J. Kruger, "Hapticwalker-A novel haptic foot device," ACM Trans. Appl. Perception (TAP), vol. 2, no. 2, pp. 166-180, Apr. 2005

[18] W. E. Ichinose, D. J. Reinkensmeyer, D. Aoyagi, J. T. Lin, K. Ngai, V. R. Edgerton, S. J. Harkema, and J. E. Bobrow, "A robotic device for measuring and controlling pelvic motion during locomotor rehabilitation," in Proc. IEEE Eng. Med. Biol. Soc. Meeting, Cancun, Mexico, Sep. 2003, pp. 1690-1693.

[19] D. Aoyagi, W. E. Ichinose, S. J. Harkema, D. J. Reinkensmeyer, and J. E. Bobrow, "An assistive robotic device that can synchronize to the pelvic motion during human gait training," in Proc. IEEE 9th Int. Conf. Rehabil. Robot., Chicago, IL, Jun./Jul. 2005, pp. 565-568.

[20] R. Baker, "Pelvic angles: A mathematically rigorous definition which is consistent with a conventional clinical understanding of the terms," Gait Posture, vol. 13, no. 1, pp. 1-6, Feb. 2001.

[21] D. Aoyagi, W. E. Ichinose, D. J. Reinkensmeyer, and J. E. Bobrow, "Human step rehabilitation using a robot attached to the pelvis," presented at the ASME Int. Mechan. Eng. Congr. Expo., Anaheim, CA, Nov. 2004.

[22] J. E. Bobrow and B. W. McDonell, "Modeling, identification, and control of a pneumatically actuated, force controllable robot," IEEE Trans. Robot. Autom., vol. 14, no. 5, pp. 732-742, Oct. 1998.

[23] F. M. Maynard, M. B. Bracken, G. Creasey, J. F. Ditunno, W. H. Donovan, T. B. Ducker, S. L. Garber, R. J. Marino, S. L. Stover, C. H. Tator, R. L. Waters, J. E. Wilberger, and W. Young, "International standards for neurological and functional classification of spinal cord injury," Spinal Cord, vol. 35, no. 5, pp. 266-274, 1997.

[24] S. J. Harkema, S. L. Hurley, U. K. Patel, P. S. Requejo, B. H. Dobkin, and V. R. Edgerton, "Human lumbosacral spinal cord interprets loading during stepping," J. Neurophysiol., vol. 77, no. 2, pp. 797-811, 1997.

[25] D. Surdilovic and R. Bernhardt, "String-man: A new wire robot for gait rehabilitation," in Proc. IEEE Int. Conf. Robot. Autom., 2004, vol. 2, pp. 2031-2036.

Daisuke Aoyagi received the B.Eng. degree in mechanical engineering from the Waseda University, Tokyo, Japan, in 1998 and the M.S. and Ph.D. degrees in mechanical and aerospace engineering from the University of California, Irvine, in 2003 and 2006, respectively.

He is currently a Research Engineer at the Los Amigos Research and Education Institute, Downey, CA. His research interests are in robot design, control, and application in rehabilitation.

Wade E. Ichinose received the B.S. and M.S. degrees in mechanical and aerospace engineering from the University of California, Irvine, in 2002 and 2005 , respectively.

He is currently a Research Engineer at Los Amigos Research and Education Institute, Downey, CA, where he began work in 2005 and started development on the second generation of the PAM/POGO device. 


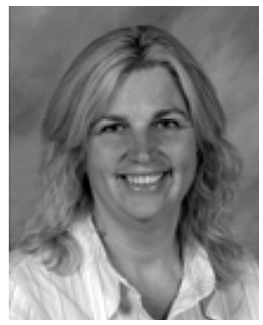

Susan J. Harkema received the B.S. and Ph.D. degrees in physiology from Michigan State University, East Lansing, and postdoctoral fellowship in neurology at the University of California, Los Angeles.

She was an Assistant Professor in the Department of Neurology and the Brain Research Institute. Currently she is an associate professor in the University of Louisville School of Medicine's Department of Neurological Surgery. She serves as Rehabilitation Director of the Kentucky Spinal Cord Injury Research Center and Director of Research at Frazier Rehab Institute. The focus of her research is to study neural mechanisms for human locomotion and the level of plasticity after neurologic injury.

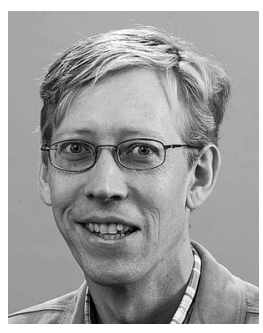

David J. Reinkensmeyer (S'93-M'93) received the B.S. degree in electrical engineering from the Massachusetts Institute of Technology, Cambridge, in 1988, and the M.S. and Ph.D. degrees in electrical engineering from the University of California, Berkeley, in 1991 and 1993, respectively.

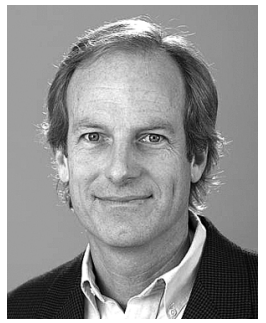

James E. Bobrow (M'85) received the Ph.D. degree from the University of California, Los Angeles, in 1983.

$\mathrm{He}$ is a Professor of Mechanical and Aerospace Engineering at the University of California, Irvine (UCI). After graduate school, he was a Senior Programmer Analyst at McDonnell Douglas Automation Company, where he developed CAM software for the Unigraphics system. He joined UCI as an Assistant Professor in 1984. While at UCI, his research interests have included optimal control and motion planning for robots, design of pneumatic actuators and sensors for automation systems, robots for rehabilitation, and machine learning systems. He has also been a Visiting Professor in Computer Science at Stanford University and in Mechanical Engineering at Massachusetts Institute of Technology, Cambridge, and he has created robots and automation devices for several startup companies, including Robomedica, Inc., and Cobra Technologies.

Prof. Bobrow is an Associate Editor of the IEEE TRANSACTIONS ON SySTEMS, MAN, AND CYBERNETICS, PART B: CYBERNETICS. He has served on the program committees or organizing committees of the leading conferences in control systems and robotics. He is currently on the egineering steering committee for Robomedica, Inc. 